\title{
Effects of age on dopamine release in the nucleus accumbens and amphetamine-induced locomotor activity in rats
}

\author{
Ruey-Ling Huang, Chih-Tien Wang, Mei-Yun Tai, Yuan-Feen Tsai*, Ming-Tsung Peng \\ Department of Physiology, College of Medicine, National Taiwan University, No. I Jen-Ai Road, Ist Section, Taipei, Taiwan (10018), ROC
}

Received 10 August 1995; revised version received 2 October 1995; accepted 2 October 1995

\begin{abstract}
The effects of age on dopamine (DA) release in the nucleus accumbens (NAc) and on amphetamine (AMPH)-induced locomotor activity were studied by microdialysis in freely-moving young ( 5 month) and old ( 24 month) rats. Both basal extracellular DA and 3,4dihydroxyphenylacetic acid (DOPAC) release and that following intra-accumbens perfusion of AMPH (1-10 $\mu \mathrm{M})$ were significantly lower in old rats. After intraperitoneal injection of AMPH $(1.5 \mathrm{mg} / \mathrm{kg})$, no age-related change in DA release was seen in the NAc, but locomotor activity was found to increase much more in young rats than in old ones. These results indicate that (1) old rats show decreased extracellular DA and DOPAC release, both in the basal state and following intra-accumbens infusion of AMPH, and (2) the age-related locomotor activity induced by systemic injection of AMPH is not paralleled by changes in DA release in the NAc.
\end{abstract}

Keywords: Microdialysis; Aging; Nucleus accumbens; Dopamine; Amphetamine; Locomotor activity

The progressive deterioration of the central dopamine (DA) system is intimately involved in motor deficits occurring during aging. Many neurochemical studies have shown that aging causes a significant decrease in striatal presynaptic DA levels and synthesis rates $[4,9,11]$. In addition, a reduced density of striatal D2 receptors has been noted in senescence [14]. It is, therefore, generally accepted that motor impairment in old animals is associated with dysfunction of the nigrostriatal DA system [3]. However, little attention has been paid to the possible effect of functional modification of the mesolimbic DA system on the changes in motor performance seen with age.

The nucleus accumbens (NAc), a forebrain component of the mesolimbic system, receives a prominent DA projection from the ventral tegmental area [17]. Decreases in both spontaneous and amphetamine (AMPH)-induced locomotor activity following lesions in the NAc have been reported [5], while direct intra-accumbens administration of DA or AMPH (a DA agonist) stimulates locomotor activity [12]. Thus, accumbens DA is thought to play a critical role in the regulation of spontaneous and

* Corresponding author. Tel.: +886 23970800 extn. 8240; fax: +886 23964350 .
AMPH-induced locomotor activity. Since locomotor activity decreases with age $[3,19]$, a decline in DA levels of the NAc might be expected in senescence. However, conflicting results for accumbens DA levels have been obtained from in vitro biochemical studies on aged animals, with some authors reporting a decrease in levels with age $[1,10]$, while others do not $[7,18]$.

AMPH is known to elicit behavioral activation, an effect considered to be mediated via the DA system [12]. The aim of the present study was therefore (1) to investigate whether aging affects the extracellular DA levels of the accumbens, and, if so, (2) to examine the effects of AMPH on this change and (3) to study the relationship between AMPH-induced locomotor activity and DA release in the NAc using microdialysis while concurrently monitoring locomotor activity in freely-moving rats.

Male Long-Evans rats, aged 5 months (young group) or 24 months (old group), were individually housed in a temperature-controlled $\left(22 \pm 1^{\circ} \mathrm{C}\right)$ room and maintained on a $12: 12 \mathrm{~h}$ light-dark cycle (lights on at $0600 \mathrm{~h}$ ) with food and water available ad libitum.

The dialysis probes were constructed as described by Lee et al. [6]. They were of concentric design, with a $2 \mathrm{~mm}$ long dialysis membrane. Each probe was tested in vitro prior to use and the recovery rates of DA and its 
Table 1

Basal extracellular monoamine concentrations in the nucleus accumbens of rats estimated from dialysate

\begin{tabular}{llll}
\hline Group & DA & DOPAC & HVA \\
\hline Young $(n=8)$ & $18.9 \pm 2.7^{*}$ & $8570.5 \pm 956.1^{*}$ & $5253.9 \pm 699.2$ \\
Old $(n=9)$ & $11.3 \pm 1.2$ & $5677.1 \pm 555.3$ & $4009.5 \pm 640.9$ \\
\hline
\end{tabular}

*Significant difference from old group, $P<0.05$. Values are corrected for probe recovery. Data are given in $\mathrm{fmol} / \mu \mathrm{l}$ and expressed as means $\pm \mathrm{SEM}$

metabolites were determined. Only those probes with a recovery rate $>10 \%$ were used in the present study.

On the day preceding a dialysis experiment, rats were anesthetized with sodium pentobarbital and ketamine. The probe was stereotaxically implanted into the right side of the NAc, using the following coordinates: A-P $+1.8 \mathrm{~mm}$ from the bregma, $\mathrm{M}-\mathrm{L}+1.8 \mathrm{~mm}$ from the midline and $\mathrm{D}-\mathrm{V}-7.5 \mathrm{~mm}$ below the dura; and secured in place with dental cement. Artificial cerebrospinal fluid (ACSF) ( $140 \mathrm{mM} \mathrm{NaCl}, 3.0 \mathrm{mM} \mathrm{KCl}, 1.0 \mathrm{mM} \mathrm{MgCl}$, $1.2 \mathrm{mM} \mathrm{CaCl}_{2}$ and $0.04 \mathrm{mM}$ ascorbate) was pumped continuously through the probe at a rate of $0.3 \mu 1 / \mathrm{min}$.

Twenty to $24 \mathrm{~h}$ after surgery, the rat was placed in an automated open-field apparatus and the perfusion rate changed to $1 \mu \mathrm{l} / \mathrm{min}$. Approximately $100 \mathrm{~min}$ later, collection of dialysis samples was initiated, with samples being taken at $20 \mathrm{~min}$ intervals, and the locomotor activity monitored throughout the whole experiment. After the collection of three baseline samples, three concentrations of $d$-AMPH sulfate (Sigma) (1,5 and $10 \mu \mathrm{M}$ in perfusate buffer) were infused consecutively at $60 \mathrm{~min}$ intervals (three $20 \mathrm{~min}$ samples), after which the perfusate was changed back to ACSF and a further six samples collected. The rat then received an intraperitoneal (i.p.) injection of AMPH $(1.5 \mathrm{mg} / \mathrm{kg})$ and five consecutive 20 min samples were collected.

The concentrations of DA, 3,4-dihydroxyphenylacetic acid (DOPAC) and homovanillic acid (HVA) in the dialysate were measured using a HPLC microbore system, coupled with electrochemical detection. Histological sectioning of the brain confirmed the accuracy of probe placement; only data from those experiments in which the probe was shown to have been placed within the NAc were analyzed.

Extracellular monoamine levels in the dialysate were expressed as the absolute concentrations after correction for probe recovery rate. Virtually no spontaneous locomotor activity was seen in either young or old rats during the behavioral test, since all experiments were conducted during the light period. Data were analyzed by the independent group $t$-test and repeated measures ANOVAs, following Scheffé's multiple comparison test. Statistical differences were defined as significant at a level of $P<0.05$.
As shown in Table 1, significantly lower levels of accumbens extracellular DA and DOPAC were seen in old rats; the decrease in extracellular HVA with age was not statistically significant. Under normal physiological conditions, the amount of extracellular DA is determined by an equilibrium between exocytotic release, uptake and a certain amount of metabolic degradation. Furthermore, the level of extracellular DOPAC has been suggested as a reliable indicator of DA terminal density [13]. Thus, the significantly lower basal extracellular levels of both DA and DOPAC observed in the present study in the NAc of old rats may suggest degeneration of the accumbens DA system with aging.

The time courses for DA and DOPAC levels following direct infusion of three concentrations of AMPH into the NAc are shown in Fig. $1 \mathrm{~A}$ and 1B, respectively. Intraaccumbens administration of AMPH produced a significant dose-dependent increase or decrease, respectively, in extracellular DA and DOPAC release. In addition, sig-
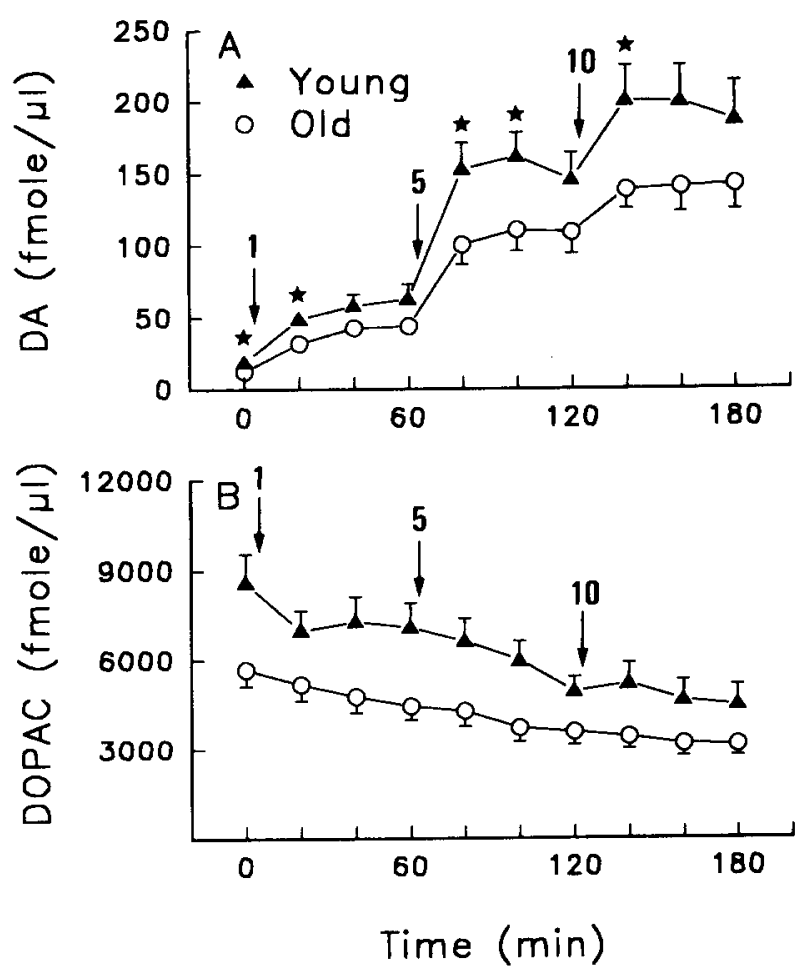

Fig. 1. Changes in NAc extracellular levels of DA (A) and DOPAC (B) in young $(n=8)$ and old $(n=9)$ rats when AMPH is added to the perfusate buffer. Three basal samples were taken before addition of AMPH; since these were all identical, only the final value (time = $0 \mathrm{~min}$ ) was used in the analysis. The addition of three increasing concentrations of AMPH $(1,5$ and $10 \mu \mathrm{M})$ is shown by arrows. Changes in DA and DOPAC levels was analyzed by repeated measures ANOVAs. A significant time effect was found for both DA and DOPAC levels $(F(9,135)=96.65$ and 31.53 , respectively; $P<0.001)$. A significant age effect was seen for DOPAC $(F(1,15)=6.91, P<0.05)$. For DA release, only the age $\times$ time effect $(F(9,135)=2.80, P<0.05)$ was statistically significant; thus subsequent post-hoc analysis was used ( ${ }^{*} P<0.05$ when compared to corresponding value for old rats). Data are the mean \pm SEM. 

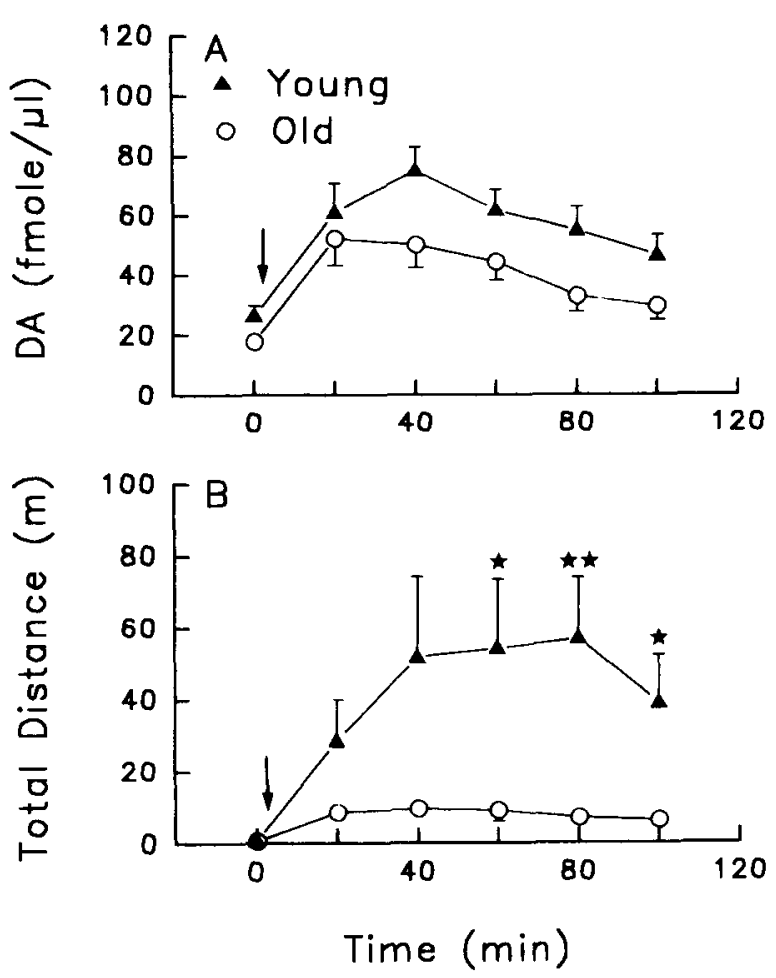

Fig. 2. Effect of intraperitoneal injection of AMPH $(1.5 \mathrm{mg} / \mathrm{kg})$ on extracellular accumbens DA release (A) and locomotor activity (B) of young $(n=8)$ and old $(n=9)$ rats. Repeated measures ANOVAs were performed on the DA level and behavioral data. A significant time effect was seen on DA and locomotor activity, with values of $F(5,75)=$ 19.47 and $7.36, P<0.001$, respectively. However, no significant age effect or age $X$ time effect was seen in the extracellular release of accumbens DA after AMPH treatment. In contrast, significant age and age $\times$ time effects were seen on locomotor activity $(F(1,15)=6.33$, $P<0.05 ; F(5,75)=4.27, P<0.01$, respectively) following AMPH treatment and subsequent post-hoc analysis was used $\left({ }^{*} P<0.05\right.$, ${ }^{* *} P<0.01$ when compared to corresponding value for old rats). The arrows indicate i.p. injection of AMPH. Data are the mean \pm SEM.

nificant age $\times$ time and age effects were found for DA and DOPAC release, respectively, after local infusion of AMPH. However, if the changes in DA and DOPAC levels were expressed as percent of basal release (data not shown), neither the DA nor the DOPAC response showed a marked age-related difference. No significant drug-induced locomotor activity was seen during intraaccumbens AMPH infusion in either age group; this probably results from the doses used, which are too low to elicit the behavioral response [12]. In addition, it is possible that unilateral perfusion of AMPH may only affect a limited region of the brain.

Since, in rats, higher doses of AMPH result in stereotyped behavior rather than locomotor activity [15], a moderate dose of AMPH $(1.5 \mathrm{mg} / \mathrm{kg})$ was chosen. A significantly increased DA release was seen in both age groups after i.p. injection. In contrast to the significant age $\times$ time effect observed following intra-accumbens AMPH infusion, no obvious age effect or age $\times$ time interaction was seen (Fig. 2A). This difference may be attributable to the fact that systemic treatment with AMPH has a more complex and divergent effect on neurotransmitter system than does local AMPH infusion. As shown in Fig. 2B, locomotor activity increased in both age groups, with the response in young rats being significantly greater.

The magnitude and temporal characteristics of the DA and DOPAC responses following AMPH treatment of young rats seen in the present study are consistent with those of certain other authors [13,16]. In addition, the absolute concentrations of extracellular DA and DOPAC in old rats following intra-accumbens AMPH infusion were lower than those in young ones, suggesting that the terminal field of the NAc DA system may degenerate with age. However, no age-related differences were seen when the data were expressed as percentage of basal release. This difference might, therefore, be explained by the lower basal release observed in the old rats, and the absence of any age-related differences in DA release (expressed as a percentage change) after AMPH challenge suggests that the mechanisms of AMPH-induced DA release in the NAC remain unchanged during the aging process.

As mentioned above, DA in the accumbens plays a critical role in the regulation of spontaneous and AMPHinduced locomotor activity $[5,12]$. Thus the decreased basal DA release seen in old rats in the present study, which indicates degeneration of the NAc DA system, may be one cause of the decline in spontaneous locomotor activity in old rats $[3,19]$. The NAc DA release elicited after i.p. injection of AMPH in young rats may be closely related to locomotor activation [16]. However, this relationship between DA release and locomotion following systemic AMPH treatment may change with age, since the AMPH-induced locomotion seen in old rats was much lower than in young ones, although there was no significant difference in extent of DA release following i.p. injection of AMPH between the two age groups. It is therefore reasonable to postulate that the amount of DA release in old rats after i.p. injection of AMPH may remain unchanged, but that the mechanisms mediating locomotion may deteriorate beyond the level of presynaptic DA release during aging. The loss of D2 receptors has been suggested to be related to the locomotor deficits of aging [14] and may explain this age-related change in AMPHinduced locomotion. Alternatively, systemic AMPH injection may also affect other NAc neurochemical systems involved in motor function, such as glutamate, acetylcholine and serotonin levels, said to change with age $[2,8,20]$. Further studies are needed to investigate these possibilities.

In summary, although old rats show decreased extracellular DA and DOPAC release, both basal and intraaccumbens-injected AMPH-induced, as well as decreased locomotor activity, no obvious age-related change in accumbens DA release was seen following systemic AMPH 
challenge, suggesting that the age-related AMPH-induced locomotor activity may not be paralleled by changes in DA release in the NAc.

This work was supported by the National Science Council Grant NSC-83-0412-B002-031, ROC.

[1] Carfagna, N., Trunzo, F. and Moretti, A., Brain catecholamine content and turnover in aging rats, Exp. Gerontol., 20 (1985) 265269.

[2] Donzanti, B.A., Hite, J.F. and Yamamoto, B.K., Extracellular glutamate levels increase with age in the lateral striatum: potential involvement of presynaptic D-2 receptors, Synapse, 13 (1993) 376-382.

[3] Emerich, D.F., Mcdermott, P., Krueger, P., Banks, M., Zhao, J., Marszalkowski, J., Frydel, B., Winn, S.R. and Sanberg, P.R., Locomotion of aged rats: relationship to neurochemical but not morphological changes in nigrostriatal dopaminergic neurons, Brian Res. Bull., 32 (1993) 477-486.

[4] Gozlan, H., Daval, G., Verge, D., Spampinato, U., Fattaccini, C.M., Gallissot, M.C., El Mestikawy, S. and Hamon, M., Aging associated changes in serotoninergic and dopaminergic pre- and postsynaptic neurochemical markers in the rat brain, Neurobiol. Aging, 11 (1990) 437-449.

[5] Koob, G.F., Riley, S.J., Smith, S.C. and Robbins, T.W., Effects of 6-hydroxydopamine lesions of the nucleus accumbens septi and olfactory tubercle on feeding, locomotor activity, and amphetamine anorexia in the rat, J. Comp. Physiol. Psychol., 92 (1978) 917-927.

[6] Lee, E.H.Y., Chang, S.Y. and Chen, A.Y.J., CRF facilitates NE release from the hippocampus: a microdialysis study, Neurosci. Res., 19 (1994) 327-330.

[7] Lee, J.M., Ross, E.R., Gower, A., Paris, J.M., Martensson, R. and Lorens, S.A., Spatial learning deficits in the aged rat: neuroanatomical and neurochemical correlates, Brain Res. Bull., 33 (1994) 489-500.

[8] Markowska, A.L., Stone, W.S., Ingram, D.K., Reynolds, J., Gold, P.E., Conti, L.H., Pontecorvo, M.J., Wenk, G.L. and Olton, D.S., Individual differences in aging: behavioral and neurobiological correlates, Neurobiol. Aging, 10 (1989) 31-43.
[9] Marshall, J.F. and Rosenstein, A.J., Age-related decline in rat striatal dopamine metabolism is regionally homogeneous, Neurobiol. Aging, 11 (1990) 131-137.

[10] Moretti, A., Carfagna, N. and Trunzo, F., Effect of aging on monoamines and their metabolites in the rat brain, Neurochem. Res., 12 (1987) 1035-1039.

[11] Morgan, D.G., May, P.C. and Finch, C.E., Dopamine and serotonin systems in human and rodent brain: effects of age and neurodegenerative disease, J. Am. Geriatr. Soc., 35 (1987) 334 345.

[12] Pijnenburg, A.J.J., Honig, W.M.M., Van der Heyden, J.A.M. and Van Rossum, J.M., Effects of chemical stimulation of the mesolimbic dopamine system upon locomotor activity, Eur. J. Pharmacol., 35 (1976) 45-58.

[13] Robinson, T.E. and Camp, D.M., The feasibility of repeated microdialysis for within-subjects design experiments: studies on the mesostriatal dopamine system. In T.E. Robinson and J.B. Justice, Jr. (Eds.), Microdialysis in the Neurosciences, Techniques in the Behavioral and Neural Sciences, Vol. 7, Elsevier, Amsterdam, 1991, pp. 189-234.

[14] Roth, G.S. and Joseph, J.A., Cellular and molecular mechanisms of impaired dopaminergic function during aging, Ann. N. Y. Acad. Sci., 719 (1994) 129-135.

[15] Schiorring, E., An open field study of stereotyped locomotor activity in amphetamine-treated rats, Psychopharmacology, 66 (1979) 281-287.

[16] Sharp, T., Zetterström, T., Ljungberg, T. and Ungerstedt, U., A direct comparison of amphetamine-induced behaviours and regional brain dopamine release in the rat using intracerebral dialysis, Brain Res., 401 (1987) 322-330.

[17] Ungerstedt, U., Stereotaxic mapping of the monoamine pathways in the rat brain, Acta. Physiol. Scand. Suppl., 367 (1971) 1-48.

[18] Watanabe, H., Differential decrease in the rate of dopamine synthesis in several dopaminergic neurons of aged rat brain, Exp. Gerontol., 22 (1987) 17-25.

[19] Willig, F., Palacios, A., Monmaur, P., M'Harzi, M., Laurent, J. and Delacour, J., Short-term memory, exploration and locomotor activity in aged rats, Neurobiol. Aging, 8 (1987) 393-402.

[20] Wu, C.F., Bertorelli, R., Sacconi, M., Pepeu, G. and Consolo, S., Decrease of brain acetylcholine release in aging freely-moving rats detected by microdialysis, Neurobiol. Aging, 9 (1988) 357361 . 